presentations; tutorials; and an equipment exhibition.

Among the plenary speakers are Nobel laureate Ada Yonath (Weizmann Institute Rehovot, Israel), presenting a lecture on "The ribosome: An energetic cellular nano machine"; Monica Olvera de la Cruz (UNAM), "Platonic and Archimedean geometries in elastic membranes"; Manish Chhowalla (Rutgers University, USA), "Solution processable two-dimensional materials for energy applications"; Carlos A. Paz de Araujo (University of Colorado, USA), "Materials science and device technology from laboratory to real world applications"; and Bao-Lian Su (University of Namur, Belgium), "Living hybrid materials for green energy production, environmental remediation and smart cell-therapy." During a Science Luncheon, Luis F. Rodríguez (UNAM) will present a talk on "Radio astronomy: The achievements and the challenges."

The list of symposia is as follows (*designates symposia co-organized by SMM and MRS).

\section{Nano Science and Technology}

1A: Low-Dimensional Bismuth-based Materials*

1B: Nanostructured Carbon Materials for MEMS/NEMS and Nanoelectronics*
1C: Nanostructured Materials and Nanotechnology

\section{Materials Characterization}

2A: The Role of Surfaces and Interfaces in Materials Processes*

2B: Novel Characterization Methods for Biological Systems*

2C: Quantitative Measurements with Atomic Force Microscopy in Fluids*

2D: Structural and Chemical Characterization of Metals, Alloys, and Compounds

\section{Materials for Energy Production}

3A: Materials for Polymer Electrolyte Membrane Fuel Cells*

3B: Photocatalytic and Photoelectrochemical Nanomaterials for Sustainable Energy*

3C: Photovoltaics, Solar Energy Materials, and Technologies

3D: New Catalytic Materials

3E: Renewable Energy and Sustainable Development

\section{Biomaterials}

4A: Nanotechnology-enhanced Biomaterials and Biomedical Devices*

4B: Biomaterials for Medical Applications

\section{Polymers}

5A: Soft Responsive Materials*
5B: New Trends in Polymer Chemistry and Characterization

\section{Electronics and Photonics}

6A: Organic Materials for Electronics and Photonics*

6B: Low-Dimensional Semiconductor Structures*

6C: Advances in Semiconducting Materials

6D: Materials and Devices for LargeArea Electronics*

\section{Fundamental Materials Science}

7A: Advances in Computational Materials Science

7B: Concrete with Smart Additives and Supplementary Cementitious Materials*

7C: NACE: Corrosion and Metallurgy

7D: Advanced Structural Materials

7E: Interfaces, Structure, and Domain Engineering in Ferroic Systems*

7F: Solid-State Chemistry of Functional Inorganic Materials*

\section{General}

8A: Strategies for Academy-Industry Relationship

For additional information on the Congress, access www.mrs-mexico.org.mx/ imrc2012.

\title{
Conference on combinatorial materials S\&T to be held in October
} http://combi2012.com

$\mathbf{T}$ he 7 th International Conference on Combinatorial Materials Science and Technology will be held on October 21-24, 2012 in Charleston, South Carolina. The workshop is devoted to combinatorial materials, attracting scientists worldwide. The conference will have representatives from academia, government laboratories, and industry. The conference website with the tentative sched- ule and names of the invited speakers are available at http://combi2012.com. The conference is endorsed by the Materials Research Society.

The focus of this workshop will be multifold. First, it aims to foster international collaboration by inviting participants from a wide variety of backgrounds. Topically, there will be an emphasis on the contributions of both computational and experimental combinatorial materials science to the fields of energy, sensing, electronic materials, and heterogeneous catalysis. Additionally, emphasis will be placed on balancing academic and industrial contributions to provide perspectives on how highthroughput methodologies are brought to bear on questions of scientific and engineering importance.

\section{wwW.mrs.org}

